\title{
Aprendizaje autodirigido en la educación superior: una perspectiva para la modalidad en línea
}

\author{
Emmanuel Moratilla Jaramillo
}

\begin{abstract}
Resumen
El objetivo de este artículo es presentar las dimensiones del aprendizaje autodirigido en la educación superior a partir de una perspectiva de modalidad en línea; las dimensiones son: la planificación del aprendizaje, el deseo de aprender, la autogestión, la autoconfianza y la autoevaluación. Aunque, teóricamente, cada dimensión del aprendizaje autodirigido es discutida por separado, en la práctica educativa están entrelazadas y la relación entre el docente y el estudiante tiende a ser más colaborativa. En conclusión, el aprendizaje autodirigido está presente en todo momento de la vida académica y personal y esto provoca que una persona con tendencia a la autodirección pueda trasladar su aprendizaje a contextos nuevos y poco familiares.
\end{abstract}

Palabras clave: aprendizaje autodirigido, educación en línea, docentes, educación superior.

\section{SELF-DIRECTED LEARNING IN HIGHER EDUCATION: A PERSPECTIVE FOR ONLINE EDUCATION}

\begin{abstract}
The aim of this article is to present the dimensions of self-directed learning in higher education through the perspective of online education. The dimensions are: learning planning, learning desire, self-direction, self-confidence and self-assessment. Even though each dimension theory of the self-directed learning is discussed separately, in the learning practice these are interrelated and the relationship between the faculty and teachers becomes more collaborative. In conclusion, self-directed learning is present in every moment of the academic and personal life allowing a person with a tendency to be self-directed to transfer his/her knowledge to new and unfamiliar contexts.
\end{abstract}

Keywords: self-directed learning, online education, faculty, higher education. 


\section{Emmanuel Moratilla Jaramillo}

e.moratilla@ebc.edu.mx

Licenciado en Psicología, titulado con mención honorífica por la Facultad de Estudios Superiores Iztacala de la Universidad Nacional Autónoma de México, UNAM. Actualmente colabora como Coordinador de Investigación Educativa en la Dirección Académica de la Rectoría de la Escuela Bancaria y Comercial. Tiene experiencia como diseñador instruccional de cursos en línea y programas académicos de licenciatura y posgrado, en coordinación de eventos académicos, en orientación metodológica y en gestión de publicaciones para congresos y revistas de estudiantes, docentes y administrativos de universidades. Su línea de investigación se centra en el análisis teórico del aprendizaje autodirigido en la educación superior. 


\section{Introducción}

El impacto de las Tecnologías de la Información y la Comunicación (TIc) ha provocado cambios en la manera de vivir, de comunicar y, sobre todo, de aprender, esto principalmente, por la rapidez que ofrecen al consultar y circular información variada y en volúmenes elevados; de ahí que la sociedad demande sistemas educativos actualizados, accesibles y flexibles. El Estudio de Educación en línea en México, con una muestra de 1128 participantes, considera que el aspecto más importante de una modalidad en línea es la flexibilidad de horarios, le sigue con $16 \%$ el costo y con $11 \%$ el reconocimiento que tenga la institución (Asociación de Internet mx, 2019).

El estudio anterior también reporta que la principal motivación de los estudiantes y de los interesados en estudiar en una modalidad en línea es la flexibilidad en el plan de estudios; además reporta que los interesados en estudiar en línea estiman que pueden invertir menos de cinco mil pesos mensualesyconsideran que bajo esta modalidad, se exige mayor responsabilidad, proactividad y dedicación (ver figura 1).

Figura 1. Aspectos que los estudiantes consideran importantes en una modalidad en línea. Fuente: elaboración propia.

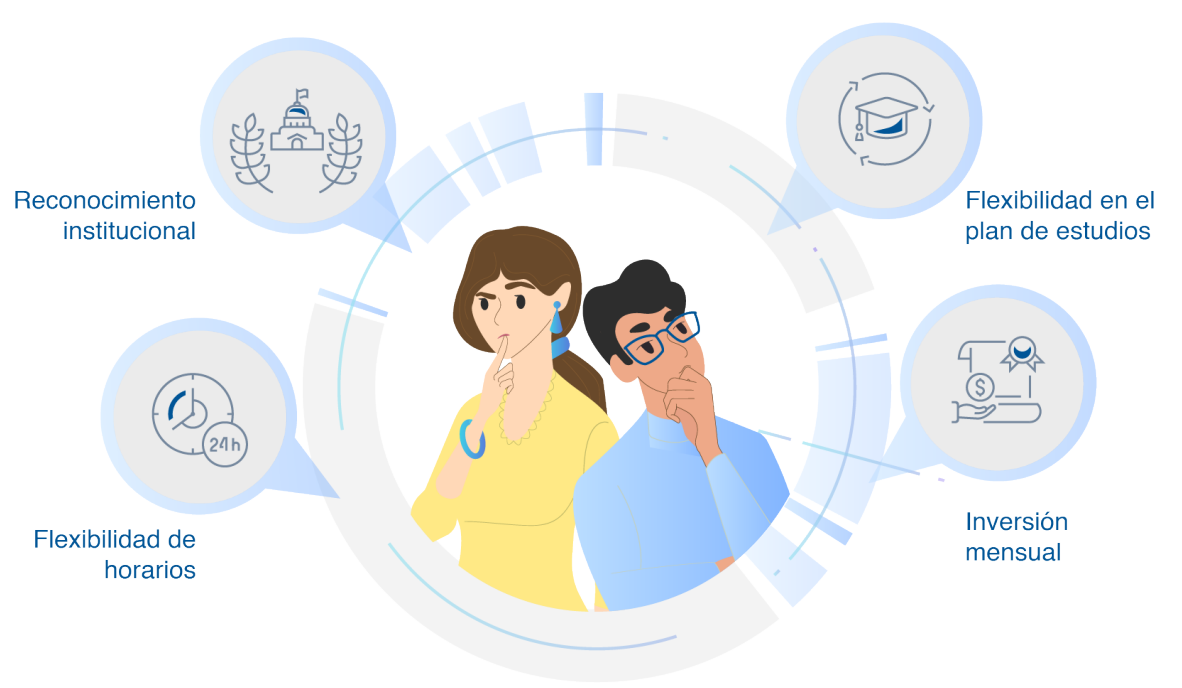

De acuerdo con Sotelo et al. (2009), un estudiante en línea además de utilizar diariamente la computadora y los programas básicos como un procesador de textos, elaborador de hoja de cálculo y de presentaciones, es crucial que se le motive e impulse en el desarrollo de habilidades de estudio antes de ingresar a un programa en línea, ya que en gran medida, esto podrá predecir el nivel de éxito del aprendizaje.

En este contexto, un concepto central en el estudio y la práctica del aprendizaje en línea es el aprendizaje autodirigido, el cual tiene su génesis en los contextos informales e independientes, lejos de una institución educativa (Cerda y Osses, 2012; Cerda y Saiz, 2015; Márquez et al., 2014). Por tal motivo, se vuelve interesante considerar al aprendizaje autodirigido como una teoría 
"Aprendizaje autodirigido en la educación superior: una perspectiva para la modalidad en línea"

Emmanuel Moratilla Jaramillo

Vol. 22, Núm. 3, mayo-junio 2021

Revista Digital Universitaria

que propicia un análisis reflexivo sobre las habilidades de estudio independiente que un estudiante puede desarrollar, más allá de la retención de la información y particularmente en los estudiantes de una modalidad en línea. Por lo tanto, el objetivo de este artículo es presentar las dimensiones del aprendizaje autodirigido en la educación superior a partir de la perspectiva de una modalidad en línea.

\section{Metodología}

Las actividades para desarrollar este artículo consistieron en:

1. La delimitación del rango de estudio, el cual fue de 2006 a 2017.

2.Una búsqueda de artículos o publicaciones en tres unidades de análisis: Google Académico, Mendeley y Elsevier-España.

3. La composición de la muestra, la cual consistió en 13 publicaciones distribuidas de la siguiente manera:

- Google Académico: 4 publicaciones

- Mendeley: 6 publicaciones

- Elsevier- España: 3 publicaciones

La muestra inicial comenzó con la revisión de la publicación de Fisher et al. (2001) respecto a un instrumento de evaluación de aprendizaje autodirigido. Después, se rastreó el uso de este instrumento en poblaciones latinoamericanas, en particular con los autores Cerda y Osses (2012) en Chile, quienes también expresaron la importancia de esclarecer el concepto de aprendizaje autodirigido; así, se dio preferencia a la línea de investigación de tales autores para la composición de este artículo.

\section{El concepto de aprendizaje autodirigido}

El aprendizaje autodirigido se define como el proceso de aprendizaje estratégico y autorreflexivo donde el estudiante toma la iniciativa, con o sin la ayuda de otros, para diagnosticar sus necesidades de aprendizaje, formular sus metas e identificar los materiales necesarios para aprender (Cerda y Osses, 2012; Flores et al., 2015 y Márquez et al., 2014); involucra una planificación, la implementación y la evaluación de sus propias experiencias de aprendizaje (Cerda y Saiz, 2015); así como la motivación para asumir la responsabilidad en la construcción de significados mediante el monitoreo de las propias cogniciones y una autogestión (García, 2012; Garrison, 1997).

Este tipo de aprendizaje se puede entender como una estrategia flexible cuando el estudiante adquiere y emplea intencionalmente habilidades de aprendizaje autodirigido en la solución de problemas o en sus demandas académicas (Velázquez et al., 2016),en otras palabras, él tiene independencia sobre lo que quiere aprender con base en sus habilidades y en las condiciones de su contexto. 


\section{Un aprendizaje multidimensional}

Las dimensiones del aprendizaje autodirigido son: la planificación del aprendizaje, el deseo de aprender, la autogestión, la autoconfianza y la autoevaluación (ver figura 2) y a continuación, se presentará cada una.

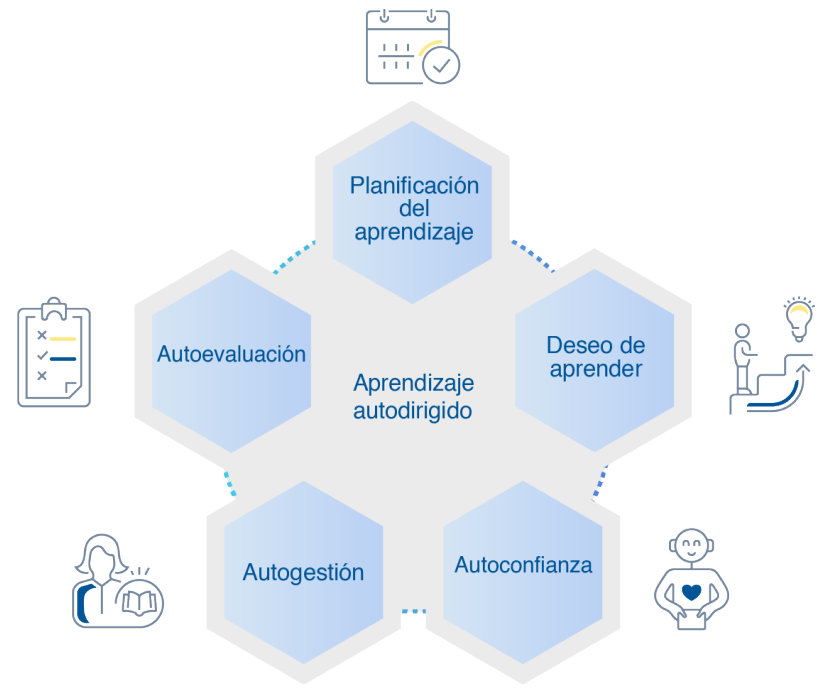

\section{La planificación del aprendizaje}

Se refiere a las capacidades de una persona para organizar y para regular sus tiempos en función de las actividades del aprendizaje (Fasce et al., 2011; Flores et al., 2015; Velázquez et al., 2016). Es recomendable que en una modalidad en línea se establezca una fecha de inicio y de término de las actividades académicas, esto para posibilitar que el estudiante organice su tiempo entre el estudio en línea y el que puede disponer para otras actividades.

\section{El deseo de aprender}

Garrison (1997) denomina a la motivación como el deseo o anhelo de una persona por aprender de manera proactiva y placentera. En recientes investigaciones, la motivación alude al interés del estudiante por conocer nuevos conceptos y a la capacidad para disfrutar del proceso (Cerda y Saiz, 2015; Fasce et al., 2011; Flores et al., 2015; Velázquez et al., 2016).

La motivación o el deseo de aprender, está influenciado por las condiciones del contexto y se trata de un mecanismo necesario para que los estudiantes asuman la responsabilidad de sus decisiones relacionadas con su aprendizaje. Esta dimensión se hace presente desde el momento en el que una persona envía sus datos para comenzar su proceso de inscripción, continua durante el estudio y finaliza cuando concluye sus estudios en una modalidad en línea. 
"Aprendizaje autodirigido en la educación superior: una perspectiva para la modalidad en línea" Emmanuel Moratilla Jaramillo

Vol. 22, Núm. 3, mayo-junio 2021

Revista Digital Universitaria

\section{La autoconfianza}

Se refiere a las características positivas que una persona se atribuye sobre su capacidad de aprendizaje, también, se relaciona con aprendizajes más profundos y con aprendizajes que implican una menor retención de hechos (Fasce et al., 2011; Flores et al., 2015; Márquez et al., 2014; Velázquez et al., 2016). En una modalidad en línea se sugiere que los mensajes de retroalimentación evoquen al dominio de competencias de los estudiantes, puede ser por los canales de comunicación que la institución tenga o por medios informales, como las redes sociales.

\section{La autogestión}

Se refiere a la capacidad que posee una persona para planificar y ejecutar sistemáticamente acciones individuales para su aprendizaje, también involucra el control sobre las tareas a través de las conductas y de las actividades donde las creencias que tiene el estudiante sobre los apoyos y los recursos con los que cuenta pueden predecir el éxito en su aprendizaje (Cerda y Saiz, 2015; Fasce et al., 2011; Garrison, 1997). Desde un punto de vista educativo, la autogestión parte de un equilibrio entre el docente y el estudiante, se trata de proporcionar un apoyo, una dirección y una referencia de estándares mínimos y necesarios para lograr el éxito académico (Fasce et al., 2011; Flores et al., 2015; Garrison, 1997).

Para que las actividades de aprendizaje fortalezcan la autogestión en una modalidad en línea se propone impulsar al estudiante a que aborde los contenidos, las metodologías, las actividades, los recursos y los sistemas de evaluación, que componen un programa académico, de manera individual y mediante la organización de sus propios tiempos, ritmos y estrategias de estudio (Ponce, 2016).

\section{La autoevaluación}

Es la capacidad de una persona para analizar críticamente su desempeño con base en criterios definidos por ella misma, se trata de promover la evaluación de los resultados para saber cuáles estrategias metacognitivas pueden volver a utilizarse y cuáles no (Flores et al., 2015; Márquez et al., 2014; Rodríguez y Espinoza, 2017; Velázquez et al., 2016).

Para una modalidad en línea, se recomienda que los estudiantes compartan e intercambien observaciones y reflexiones sobre su propio desempeño, ya que estas acciones les permitirán verificar sus prácticas para la elaboración de sus tareas académicas y principalmente, su nivel de aprendizaje logrado.

\section{Conclusiones}

El aprendizaje autodirigido es un constructo que depende del objetivo y del interés del estudiante. De manera que se debe fomentar en ellos la capacidad de evaluar sus necesidades de aprendizaje para poder definir, de manera independiente, los objetivos que persiguen. 
"Aprendizaje autodirigido en la educación superior: una perspectiva para la modalidad en línea" Emmanuel Moratilla Jaramillo

Vol. 22, Núm. 3, mayo-junio 2021

Revista Digital Universitaria

Aunque, teóricamente, cada dimensión del aprendizaje autodirigido es discutida por separado, en la práctica, están fuertemente entrelazadas y la relación del docente con el estudiante es más colaborativa que aislada. Esto significa que el aprendizaje es un proceso dependiente de retroalimentaciones internas, por parte del estudiante, y externas, por parte del contexto, así, este último, se vuelve indispensable para el aprendizaje (Flores et al., 2015; Garrison, 1997; Velázquez et al., 2016).

Una persona que se autodirige en un área específica debe poseer cierto nivel de conocimiento, por lo que Fisher et al. (2001) mencionan que una persona altamente autodirigida en matemáticas puede no poseer la misma autodirección

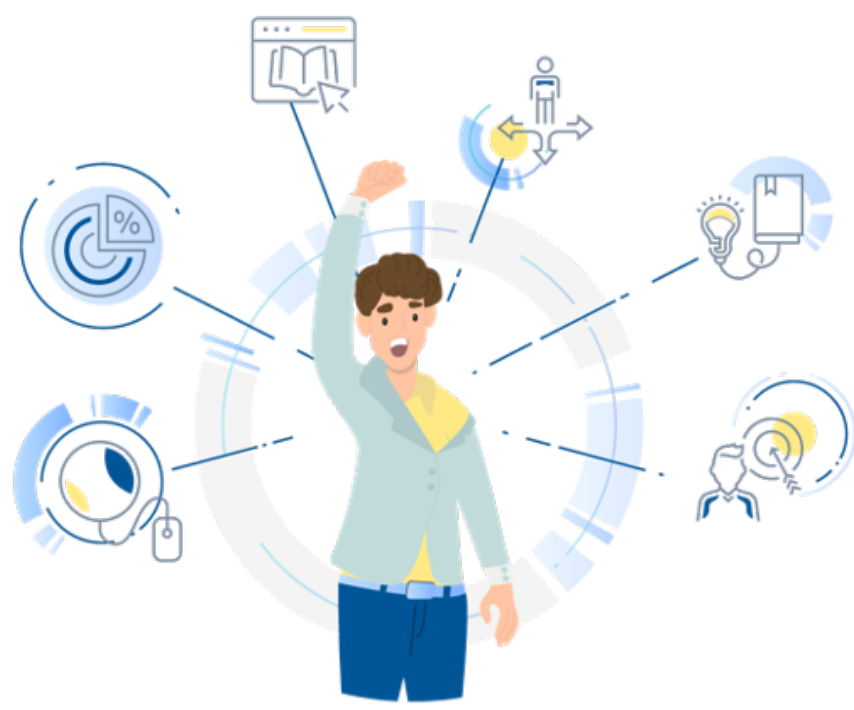
para aprender inglés. También, estudios recientes como el de Márquez et al. (2014) mencionan que la planificación es una característica perteneciente a un estilo teórico de aprendizaje de los estudiantes de medicina, quienes muestran un desarrollo del pensamiento secuencial, con alta capacidad de integración de hechos dispares en teorías coherentes.

De igual manera, el trabajo de Flores et al. (2015) reportaron, con estudiantes de psicología, que en promedio, la planificación del aprendizaje está en un nivel menor a todas las demás dimensiones. Y por su parte, Rodríguez y Espinoza (2017) informan que los estudiantes de nivel superior muestran una mayor habilidad para trazar metas sobre lo que desean alcanzar con el aprendizaje.

Figura 3. El aprendizaje autodirigido en la vida académica y personal. Fuente: elaboración propia
En conclusión, es posible que el aprendizaje autodirigido esté presente en todo momento de la vida académica y personal, y logra que una persona que posea una fuerte tendencia a la autodirección pueda trasladar su aprendizaje a contextos nuevos y poco familiares (ver figura 3). De manera particular, en una modalidad en línea es necesario impulsar las capacidades de aprendizaje autodirigido ya arraigadas en el estudiante para que conformé un patrón de desarrollo profesional y para que se sostenga y evolucione acorde a los nuevos recursos tecnológicos disponibles.

\section{Referencias}

* Asociación de Internet mx. (2019). Estudio de Educación en línea en México 2018. occMundial.https://www.asociaciondeinternet.mx/es/component/remository/ func-startdown/96/lang,es-es/?ltemid=.

* Cerda, C., y Osses, S. (2012). Aprendizaje autodirigido y aprendizaje autorregulado: Dos conceptos diferentes. Revista Médica de Chile, 140(11), 1504-1505. https://doi. org/10.4067/S0034-98872012001100020 
"Aprendizaje autodirigido en la educación superior: una perspectiva para la modalidad en línea" Emmanuel Moratilla Jaramillo Vol. 22, Núm. 3, mayo-junio 2021 Revista Digital Universitaria

* Cerda, C., y Saiz, J. L. (2015). Aprendizaje autodirigido en estudiantes de pedagogía chilenos: Un análisis psicométrico. Suma Psicológica, 22(2), 129-136. https://doi. org/10.1016/j.sumpsi.2015.08.004.

* Fasce, E., Pérez, C., Ortiz, L., Parra, P. y Matus, O. (2011). Estructura factorial y confiabilidad de la escala de aprendizaje autodirigido de Fisher, King \& Tague en alumnos de medicina chilenos. Revista Médica de Chile, 139(11), 1428-1434. https:// doi.org/10.4067/S0034-98872011001100006.

* Fisher, M., King, J. y Tague, G. (2001). Development of a self-directed learning readiness scale for nursing education. Nurse Education Today, 21(7), 516-525. https://doi.org/10.1054/nedt.2001.0589.

* Flores, J., Velázquez, B.y Salado, M. (2015). Aprendizaje autodirigido desde la educación basada en competencias en estudiantes universitarios. Tlamati, 6(1), 240-246.

* García, M. (2012). La autorregulación académica como variable explicativa de los procesos de aprendizaje universitario. Profesorado. Revista de Currículum y Formación de Profesorado, 16(1), 203-221. https://www.redalyc.org/articulo. oa?id=56724377012

* Garrison, D. R. (1997). Self-Directed Learning:Towarda Comprehensive Model.Adult Education Quarterly, 48(1), 18-33. https://doi.org/10.1177/074171369704800103

* Márquez, C., Fasce, E., Pérez, C., Ortega, J., Parra, P., Ortiz, L., Matus, O. e Ibáñez, P. (2014). Aprendizaje autodirigido y su relación con los estilos y estrategias de aprendizaje en estudiantes de medicina. Revista Médica de Chile, 142(11), 14221430. https://doi.org/10.4067/S0034-98872014001100009.

* Ponce, M. (2016). La autogestión para el aprendizaje en estudiantes de ambientes mediados por tecnología. Diálogos sobre educación, 12(7), 1-23. https://doi. org/10.32870/dse.v0i12.258.

* Rodríguez, R. y Espinoza, L. (2017). Trabajo colaborativo y estrategias de aprendizaje en entornos virtuales en jóvenes universitarios. Revista Iberoamericana para la investigación y el desarrollo educativo, 7(14), 1 - 23. http://dx.doi.org/10.23913/ride.V7i14.274.

* Sotelo, M., Ramos, D. y Tánori, A. (2009). Habilidades y actitudes en estudiantes que cursan materias en modalidad virtual-presencial en una institución de educación superior. Memorias del X Congreso Nacional de Investigación Educativa. Área temática 1: Aprendizaje y desarrollo humanos. http://www.comie.org.mx/ congreso/memoriaelectronica/v10/pdf/area_tematica_01/ponencias/0978-F.pdf

* Velázquez, B., Flores, G., Domínguez, J.y Moreno, T. (2016). Aprendizaje autodirigido y estrategias de aprendizaje en estudiantes universitarios. Foro de Estudios Sobre Guerrero, 2(3), 690-697.

\section{Cómo CITAR ESTE ARTículo}

- Moratilla Jaramillo, Emmanuel. (2021, mayo-junio). Aprendizaje autodirigido en la educación superior: una perspectiva para la modalidad en línea. Revista Digital Universitaria (RDU), 22(3). http://doi.org/10.22201/cuaieed.16076079e.2021.22.3.11 